\section{Optimism about oxytocin}

\section{By Lev Osherovich, Senior Writer}

University of California, Los Angeles researchers have evidence of a link between psychological disposition and a receptor for the neuroactive hormone oxytocin. ${ }^{1}$ A new company-Pastorus Pharma LLC-thinks the findings provide additional proof of concept for targeting the oxytocin receptor in behavioral and psychiatric indications.

A team led by Shelley Taylor, professor of psychology at UCLA, was looking for genetic factors that underlie variation in three psychological traits that influence an individual's ability to cope with stress: optimism, self-esteem and sense of mastery.

"We know that these factors affect response to stressful life events, which have a major role in the development of psychiatric disorders, including
"We knew there was a pathway [involved in stress coping], but we didn't know what it was. It was a bit of a surprise that it turned out to be oxytocin, which has previously been thought of as being involved in social or interpersonal pathways." -Shelley Taylor, University of California, Los Angeles turned out to be oxytocin, which has previously been thought of as being involved in social or interpersonal pathways."

\section{On the bright side}

ncovering the molecular basis for Taylor's findings will be tricky, as the to tackle experimentally.

One possibility is that the A version of OXTR has a lower level of function and thus is less responsive to normal levels of oxytocin compared with the $\mathrm{G}$ allele. An opposite scenario-in which the A allele responds more strongly to the hormone than the G allele-also is possible. Because levels of OXTR expression can fluctuate in response to the hormone, Taylor thinks it is too soon to guess how the receptor variants affect the protein's function.

"We really don't know if the A allele is a gain or loss of function," said Hurlemann.

He thinks that to conclusively link Taylor's findings to disease, it will be necessary to screen for OXTR polymorphisms in patients with clinically defined psychiatric disorders.

"This study was done in healthy volunteers," said Hurlemann. "You would have to investigate the oxytocin receptor polymorphism in patients" with depression, anxiety or PTSD.

Hurlemann, Cornett and Taylor noted that previous studies have examined the relationship between plasma levels of oxytocin and major depression, anxiety disorder and post-traumatic stress disorder," said René Hurlemann, associate faculty member in the Department of Psychiatry at the University of Bonn.

Other researchers had previously found that genetic variation in the oxytocin receptor (OXTR) affected self-assessed psychological well-being. ${ }^{2}$ Taylor's team examined the relationship between OXTR genotype and measurable characteristics such as dispositional optimism and depressive symptoms.

Among 326 healthy volunteers, heterozygous and homozygous carriers of the A allele of OXTR were rated lower on scales of optimism $(p=0.031)$, mastery $(p=0.043)$ and self-esteem $(p=0.004)$ than individuals homozygous for the $\mathrm{G}$ allele. About two-thirds of the subjects were A allele heterozygotes or homozygotes.

Taylor's study is among the first to identify "a specific marker for these personality traits," said Pastorus CEO and principal Glenn Cornett.

The results were reported in the Proceedings of the National Academy of Sciences.

The connection between OXTR variation and coping with stress could open a new therapeutic opportunity for modulating oxytocin signaling, which is better known as a regulator of interpersonal trust and maternal bonding. Indeed, a generic injectable form of oxytocin is used to induce delivery and promote lactation.

"We knew there was a pathway [involved in stress coping], but we didn't know what it was," said Taylor. "It was a bit of a surprise that it psychiatric disorders, but results of those studies have been mixed and are difficult to interpret because of the hormone's short half-life in the blood.

If the A allele of OXTR turns out to decrease the receptor's signaling ability, there could be a case for giving oxytocin to depressed patients.

Hurlemann said oxytocin already has been tried as an adjunct to psychological counseling in small-scale, investigator-initiated studies in autism, schizophrenia and social anxiety. He is running a study of oxytocin to alleviate symptoms of opiate withdrawal.

"Oxytocin has been considered for everything from psychiatric disorders to sleep apnea," said Daniel Jacobs, founder, chairman and CEO of Trigemina Inc., which is developing oxytocin therapeutics for pain.

Although some prior studies hinted at improvements in mood and affect from intranasal oxytocin, none was sufficiently powered to allow strong conclusions. ${ }^{3}$

Hurlemann said oxytocin therapy appears to alleviate delusional ideation in schizophrenia and reduce amygdala activation in patients with social anxiety, "but the results for depression haven't been so successful."

If the two OXTR alleles vary in their response to the hormone, it also may be useful to stratify patients in oxytocin trials by their OXTR allele.

Cornett noted that small-scale studies suggest oxytocin can alleviate social anxiety in some patients but not others. Taylor's findings could potentially account for differences in response.

"I would be very interested in genotyping responders and nonresponders" to oxytocin, said Cornett. 


\section{ANALYSIS}

\section{TARGETS \& MECHANISMS}

He added: "There could be a decent amount of overlap" between the three psychological traits measured by Taylor and the psychological risk factors in autism and schizophrenia, for which Pastorus is developing an intranasal formulation of oxytocin.

Cornett said Pastorus has a formulation and delivery technology that improves the bioavailability of nasal oxytocin.

"In comparison to the currently marketed delivery system, our technology increases bioavailability significantly. Based on results thus far, dosage consistency seems to be enhanced materially as well," said Cornett. "Our dry-powder technology involves mixing the active ingredient into cellulose particles, which then adhere to the nasal mucosa longer than do the saline drops administered in the current nasal-spray technology."

The company plans to run a dose-ranging Phase I study in "a couple hundred patients" and then a Phase IIb study in autism and schizophrenia, Cornett said. Pastorus is raising money and does not have a timeline for starting the trials.

Cornett and an undisclosed partner are the investors in Pastorus. The company is seeking additional seed funding from individual investors. Cornett said he is open to seeking venture capital financing in the future.

Trigemina has completed a 40-patient Phase II trial of TI-001, an intranasal formulation of oxytocin for chronic headache. In that trial, $50 \%$ of patients receiving oxytocin reported a decrease in headache 4 hours postdosing compared with $12 \%$ of placebo patients. However, in a subsequent 20 -patient dose-escalation study, $70 \%$ of patients receiving placebo also reported reduced pain.

Jacobs attributed the high placebo response rate to the environment in which dosing was conducted. "Whose headache doesn't go away when you're in a nice, quiet, dimly lit room?" he said.

The company plans to run a second Phase II trial next year using an improved protocol with a different dosing environment and quantitative pain endpoints.

Taylor did not patent her discoveries.

Osherovich, L. SciBX 4(37); doi:10.1038/scibx.2011.1031

Published online Sept. 22, 2011

\section{REFERENCES}

1. Saphire-Bernstein, S. et al. Proc. Natl. Acad. Sci. USA; published online Sept. 6, 2011; doi:10.1073/pnas.1113137108

Contact: Shelley E. Taylor, University of California, Los Angeles, Calif. e-mail: taylors@psych.ucla.edu

2. Lucht, M.J. et al. Prog. Neuropsychopharmacol. Biol. Psychiatry 33, 860-866 (2009)

3. MacDonald, K. \& MacDonald T.M. Harv. Rev. Psychiatry 18, 1-21 (2009)

COMPANIES AND INSTITUTIONS MENTIONED

Pastorus Pharma LLC, Laramie, Wyo.

Trigemina Inc., Mountain View, Calif.

University of Bonn, Bonn, Germany

University of California, Los Angeles, Calif. 